\title{
Reabsorción radicular de incisivos centrales por impactación de caninos. Una solución ortodóncica
}

\begin{abstract}
Tuesta O, Morzán E, Cabrejos S, Estrada M. Reabsorción radicular de incisivos centrales por impactación de caninos. Una solución ortodònciea. Rev Estomatol Herediana 2003:13(1-2) : $40-44$
\end{abstract}

\section{RESUMEN}

La impactación del canino superior permanente es un problema clínico que puede provocar una serie de secuelas que van desde la perrida de espacio en el arto para su correcta erupción, hasta la reabsorción radicular de dientes vecinos. En el presente reporte se describe el easo de un paciente con ausencia clinica de caninos superiores, que se encontraban impactados con las coronas orientadas hacia vestibular y las raices hacia palatino, produciendo reahsorción de $2 / 3$ radiculares de los incisivos centrales superiores. Ambos incisivos centrales superiotes fueton extraidos junto con la exposiciôn quinùrgiéa y posterior traceión ortodónticu do los canmos impactados. En el maxilar inferior se realizaron las extracciones de los incisivos laterales. Diez meses fueron necesarios para observar elinieamente las piezas impactadas, El tiempo de tratamiento fue de dos antos y euatro meses. Se recontomearon las cúspides de los caninos y se añadió resina compuesta para reconstruir ol borde incisal

Palabras clave: Caninos impactidos - Reabsorción nadieular - Traceión ortodóntica.

Upper central incisors roat resorption by canine impaction: an orthodontic approach ABSTRACT

Upper canine impaction is a condition capable of promoting a wide variety of clinical sequels ranging from arch length discrepancy to adjacent teeth root resorption. This report presents a case in which both upper canines were elinically absent and resorbing $2 / 3$ of upper central incisor roots. Both central incisors wend drawn and camine crowns exposed. Two buttons were bonded. Canines were orthodontically retracted. Lateral incisors were removed in mandibular arch in order to establish an acceptable occlusion. 10 months wero needed to observe both canines. Total treatmeat time was two years and four months. Cunine tips were drilled and contoured with composite.

Key words: Canine impaction - Root resorption - Retraction.
Orlando Tuesta D.'

Eduardo Morzán V.

Sandra Cabrejos ${ }^{2}$

Marco Estrada ${ }^{2}$

Proteser del Proprama de Fopecialuacion or Ortiodoncis

Ex-rasidertos del Prograna da Espocialzacion en Onodoncia Facultad de Estomatologia Urikarsiisd Penuana Cayotino Horeda

\section{Introducción}

Se conoce como dientes impactados a aquellos que se han formado dentro del hueso pero que han fracasado en el proceso de erupción (1). Cualquier pieza dentaria puede encontrarse impactada pero la mayor frecuencia de impactación se observa en terceros molares inferiores y superiores, seguidos por caninos superiores, segundos premolares superiores e inferiores y los incisivos centrales superiores (2).

La incidencia de impactación de caninos superiores varía entre $0.92 \%$ a $2.2 \%(3,5,6,7)$. En relación al sexo es dos veces más común en mujeres $(1.17 \%)$ que en varones $(0.51 \%)$ (4). La incidencia de impactación por palatino excede a la vestibular en una proporción de por lo menos 3:1 (8). El 8\% de todas las impactaciones caninas ocurre bilateralmente.

La etiología de la impactación canina es multifactorial y difícil de definir. Rara vez ocurre en forma aislada ya que es común observarla acompañada con agenesia o anomalías en forma, de incisivos laterales, con dientes deciduos retenidos, con dientes supernumerarios o con una deficiencia esquelética premaxilar. Hay que destacar que los caninos superiores están predispuestos a quedarse incluidos por presentar el periodo más largo de desarrollo, además de la vía de erupción más extensa y tortuosa $(9,10)$.

Diversos factores locales y generales están relacionados con la impactación de caninos superiores. Retención prolongada o pérdida prematura del canino deciduo, discrepancia dentoalveolar, posición anormal del germen, quis- tes o neoplasias, agenesia o alteración en la forma de incisivos laterales, hendidura alveolar, dilaceración radicular, anquilosis, trauma, cierre prematuro apical y condiciones idiopáticas son factores locales reconocidos $(4,10,11$, $12,13,14,15,16,17,18,19,20)$. Entre los factores generales se describen las deficiencias endocrinas como hipotiroidismo e hipopituitarismo, síndromes como disostosis cleidocraneal y craneosinostosis, deficiencia de vitamina $D$, presión muscular anormal, factores genéticos e irradiación $(4,11,12,13,18$, 21).

El diagnóstico y tratamiento generalmente implica un abordaje multidisciplinario donde intervienen el odontopediatra, cirujano maxilofacial, periodoncista, ortodoncista y rehabilitador (4). 
Se reporta un caso de caninos superiores impactados que habían producido severa reabsorción de incisivos centrales.

\section{Reporte del caso}

Se trata de un paciente de sexo femenino de 13 años 7 meses de edad que acude al Servicio de Poistgrado.de ortodoncia de la Facultad de estomatología de la Universidad Peruana Cayetano Heredia refiriendo que "sus dientes están desalineados" y sus dientes incisivos centrales se mueven.

\section{Examen Clínico}

Se observa una paciente mesocéfala, mesofacial, con simetría facial, con tercios faciales proporcionados, competencia labial, perfil ligeramente convexo y normodivergente (Fig.1A). El examen de la oclusión revela una relación molar derecha clase II e izquierda clase I, relaciones caninas no registrables; sobrepase horizontal $2 \mathrm{~mm}$. y sobrepase vertical $20 \%$. Los arcos dentales son ovala- dos con apiñamiento superior de $2 \mathrm{~mm}$ e inferior de $10 \mathrm{~mm}$. La línea media infe rior se encuentra desviada $1 \mathrm{~mm}$. a 1 izquierda, se evidencia ausencia clínic de piezas 13 y 23 y movilidad dentari grado 2 en piezas 11 y 21 (Fig. 1B).

\section{Exámenes Auxiliares}

-La radiografía periapical ántero su perior muestra la impactación de las pie zas 13 y 23 que producían reabsorció de aproximadamente $2 / 3$ radiculares $d$ los incisivos centrales superiore
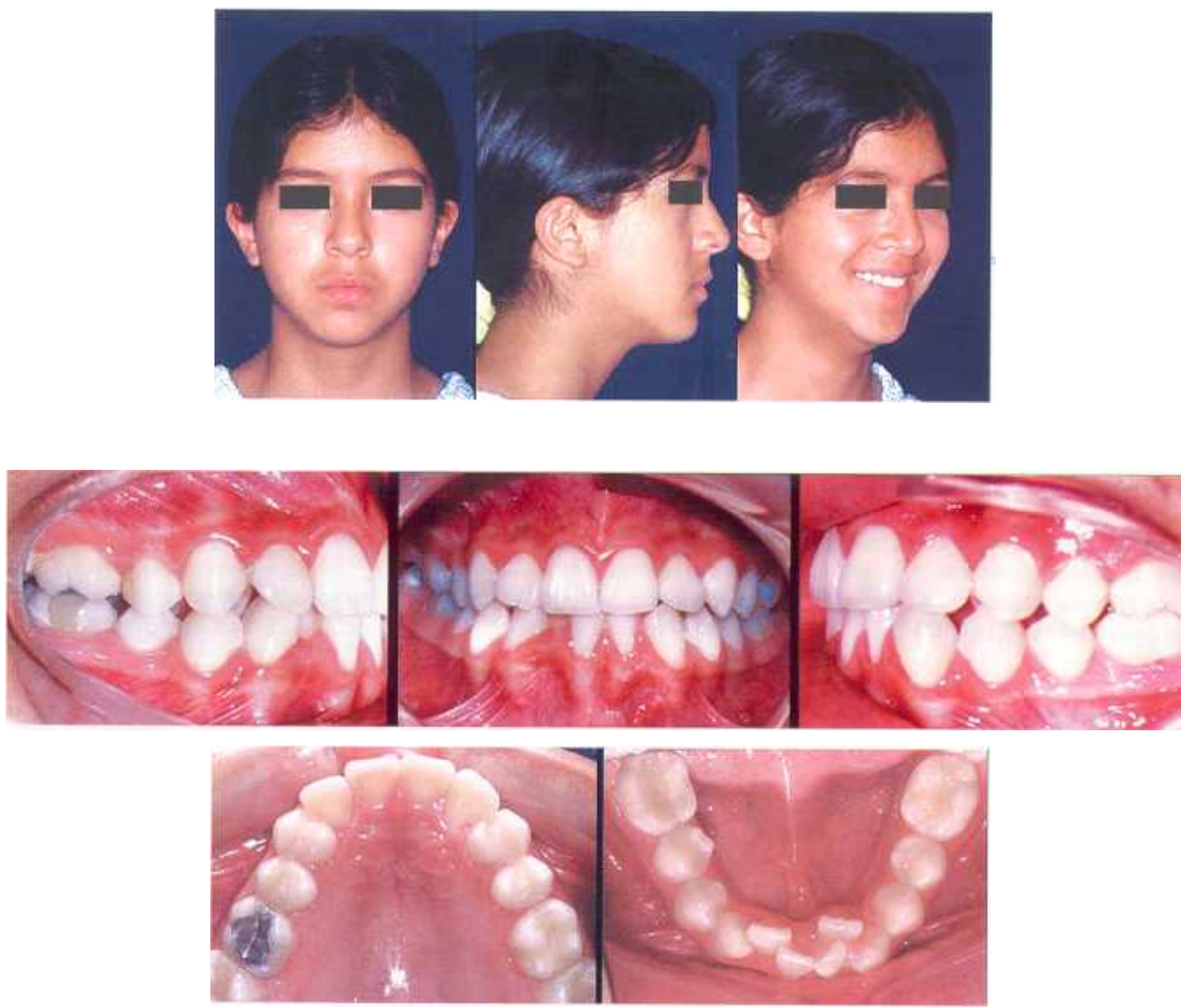

Fig. 1. Fotografias iniciales. 1A. Vistas extraorales. 1B. Vistas intraorales. 
(Fig. 2A). Según la técnica de desplazamiento horizontal de Clark, ambas piezas se encuentran con la porción coronal orientada hacia vestibular y la radicular hacia palatino. En la radiografía panorámica se observan estructuras óseas normales, 32 piezas dentarias permanentes, impactación de las piezas 13 y 23 con formación completa de raíz e inclinación coronaria mesial, reabsorción de $2 / 3$ radiculares de incisivos centrales superiores, así como tratamiento de conductos en las piezas 15 y 46 (Fig. 2B). Cefalométricamente existía una relación esquelética Clase I con birretrusión maxilar, incisivos superiores palatinizados y retruídos e incisivos inferiores lingualizados (Fig. 2C). El análisis de modelos mostró una discrepancia alveolo dentaria (DAD) superior de $-18 \mathrm{~mm}$. e inferior de $-13 \mathrm{~mm}$.

\section{Diagnóstico Definitivo}

Maloclusión Clase I con impactación de caninos superiores localizados a nivel apical de incisivos centrales superiores, con reabsorción radicular severa de incisivos centrales superiores, relación molar derecha clase II y línea media inferior desviada $1 \mathrm{~mm}$. a la izquierda.

\section{Plan de tratamiento}

Extracciones de incisivos centrales superiores, exposición quirúrgica y colocación de botones para tracción ortodóntica de caninos impactados. Extracciones de los incisivos laterales inferiores. Tratamiento ortodóncico con técnica estándar utilizando brackets arco de canto ranura $0.022 \times 0.028$ ".

\section{Tratamiento}

En una sola sesión se extrajeron los incisivos centrales superiores, se expusieron los caninos superiores y se cementaron botones a las coronas de los caninos (Fig. 3). En los incisivos extraídos se removieron los restos pulpares y se obturaron sus cámaras con cemento de ionómero vítreo. Con la finalidad de preservar la estética estos incisivos fueron adheridos con resina compuesta a un dispositivo de anclaje tipo botón de Nance asociado a un arco traspalatal fijo que fue cementado a las primeras molares (Fig. 4). En el maxilar inferior se extrajeron los incisivos laterales inferiores. Se utilizó cemento de ionómero vítreo modificado con resina para la cementación de brackets.

El alineamiento y nivelación del arco dental inferior se realizó siguiendo una mecánica convencional. En la arcada superior se utilizaron arcos de alambre seccionados para nivelación hasta la instalación de un arco de sección rectangular continuo (Fig. 5A). Los caninos fueron traccionados inicialmente con hilo elástico desde el botón de Nance y posteriormente desde el arco traspalatal. Después de 10 meses de tracción elástica se cementaron brackets a los caninos y se instalaron alambres seccionados tipo brazo de palanca "cantilever" desde las primeras molares hasta los caninos (Fig. 5B). Los incisivos centrales fueron desgastados progresivamente para generar espacio para la erupción de los caninos. Posteriormente se removió el dispositivo de anclaje superior y se colocó un arco de activación térmica (Fig. 5C).
Después de 2 años y 4 meses de iniciado el tratamiento se retiraron los brackets. Luego se recontornearon las cúspides de los caninos y mediante el uso de resina se modificó la forma de estos dientes para semejarlos a incisivos centrales. Adicionalmente se solicitó la evaluación de la condición gingival de estas piezas (Fig. 6).

Para la fase de contención se utilizaron placas de contención continuas removibles.

\section{Discusión}

La impactación canina puede provocar una serie de secuelas como (4): malposición vestibular o palatina del diente impactado, migración de dientes vecinos y pérdida de la longitud de arco, reabsorción externa del diente impactado así como de dientes vecinos, reabsorción interna, formación de quiste dentígero, infección relacionada con la erupción parcial de la pieza y dolor referido.

Aproximadamente en $0.71 \%$ de niños entre 10 a 13 años los incisivos permanentes son reabsorbidos por erupción ectópica de caninos sủperiores (22). Ericson (23) reportó reabsorción de la raíz del incisivo lateral en $12 \%$ de una muestra que presentaba caninos impactados Si bien la impactación canina no necesariamente genera reabsorciones radiculares, debido al elevado potencial de complicaciones, es prudente observar de cerca el desarrollo y erupción de estos dientes (4).

El diagnóstico de, impactación debe basarse en un examen clínico y radiográfico (2). Se han sugerido diversos
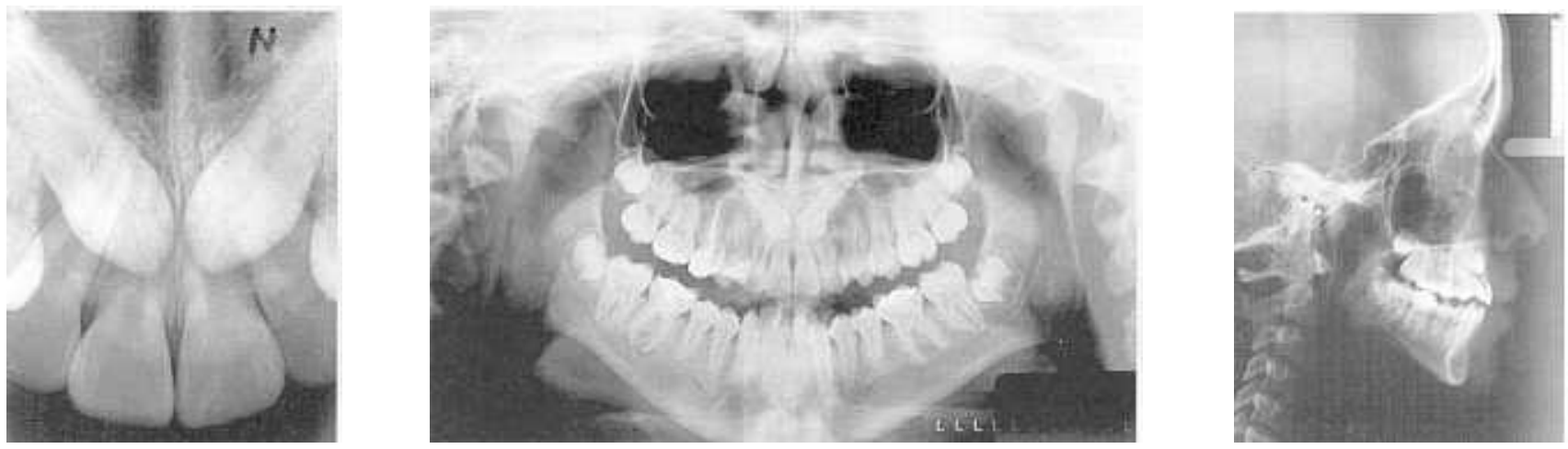

Fig. 2. Radiografias iniciales. $2 \mathrm{~A}$. Radiografía periapical anterosuperior. $2 \mathrm{~B}$. Radiografía panoramica. 2C. Radiografía cefalométrica. 


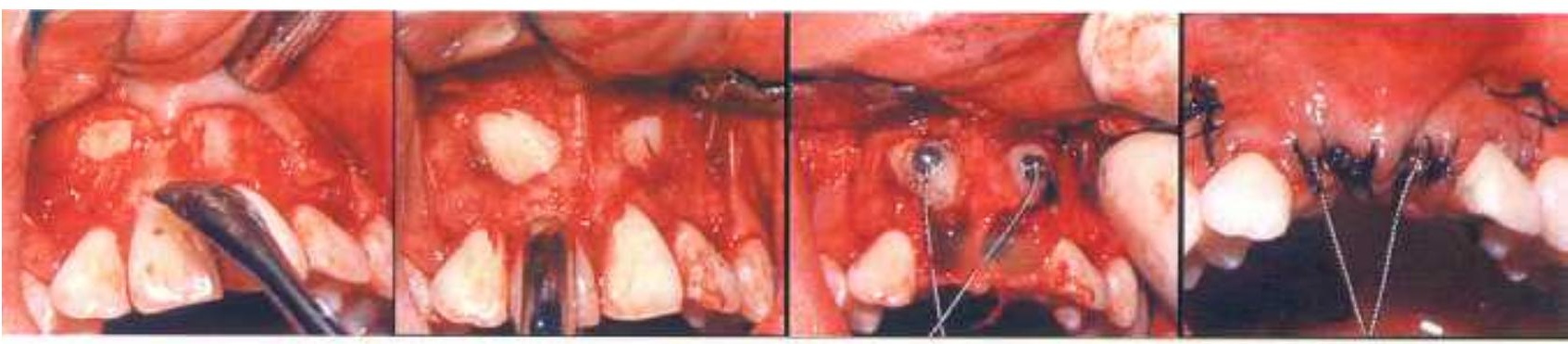

Fig. 3. Exposición quirúrgica de caninos superiores.
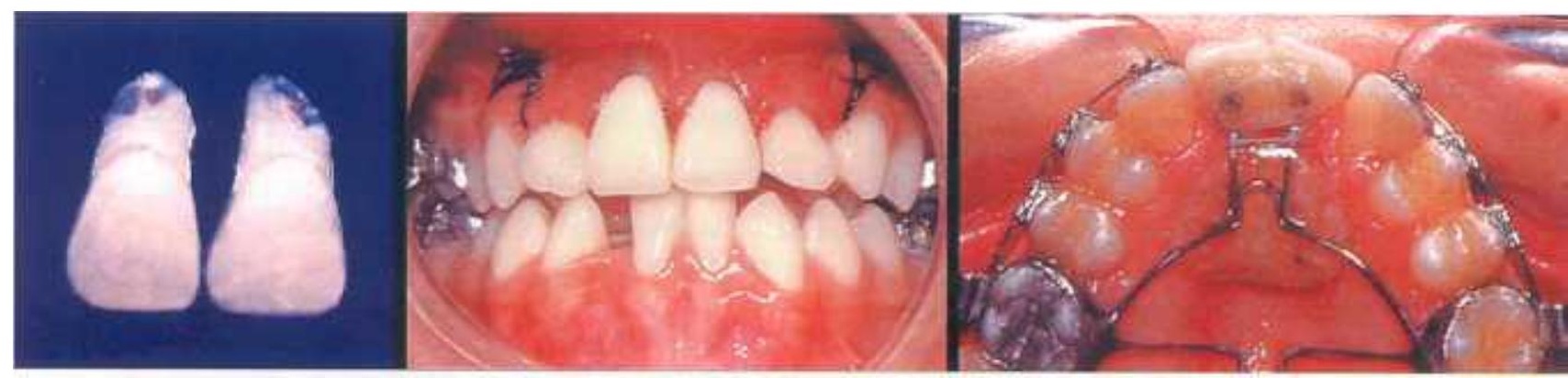

Fig. 4. Dispositivo de anclaje con incisivos adheridos con resina.
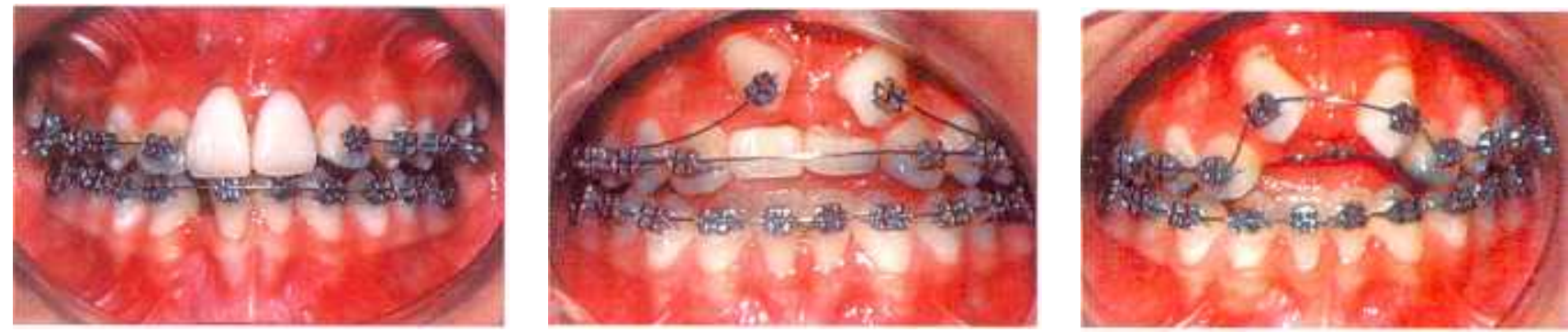

Fig. 5. Avance del tratamiento. 5A. Arcos superiores. 5B. Cantilevers en caninos. 5C. Arco de activación térmica.
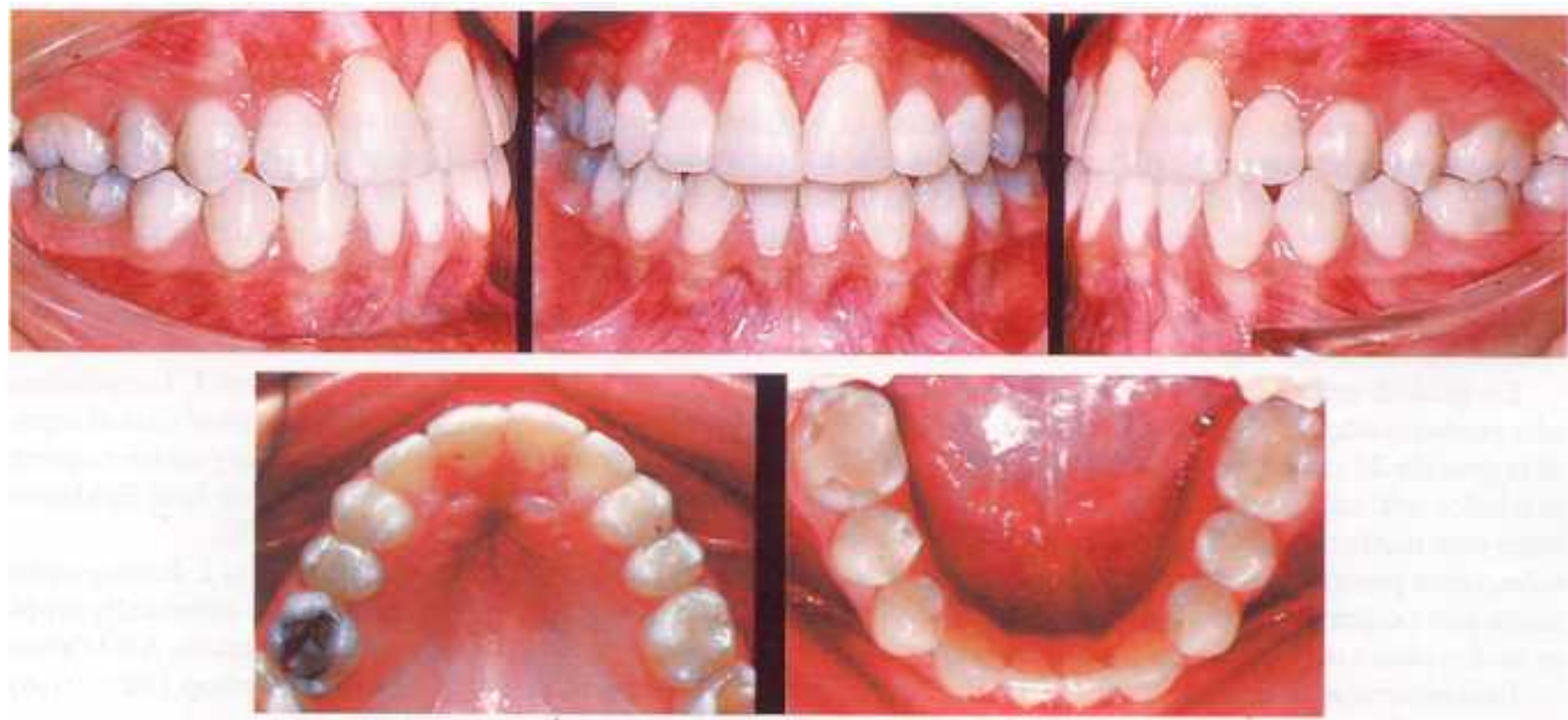

Fig. 6. Fotografias finales. 
signos clínicos como indicativos de impactación canina: erupción retrasada del canino permanente, retención prolongada del canino deciduo después de los 14 años de edad, ausencia de prominencia canina o imposibilidad de localizar la posición canina a través de palpación intraoral del proceso alveolar, presencia de una prominencia asimétrica, presencia de una prominencia palatina e inclinación distal o migración del incisivo lateral.

La ausencia de prominencia canina a edad temprana no debe ser considerada como indicativo de impactación canina. Este dato fue corroborado por Ericson (22) en un estudio realizado en 505 escolares entre los 10 a 12 años de edad donde demostró que en el $29 \%$ de los casos no se palpaba la prominencia canina a los 10 años de edad, a los 11 años no se palpaba en el 5\% de los casos y que a mayor edad no era palpable en el 3\% de los casos. Estos datos permiten afirmar que un adecuado diagnóstico de impactación canina debe incluir necesariamente una evaluación radiográfica.

La localización precisa del canino impactado es determinante, para realizar el procedimiento quirúrgico que permita la aplicación de fuerzas ortodónticas.

Según Ericson y Kurol (23) con el uso de las radiografías periapicales el clínico puede evaluar la posición del canino con suficiente exactitud en el $92 \%$ de los casos. Las radiografías periapicales permiten observar la relaçión del canino con sus dientes vecinos tanto en sentido horizontal como vertical. Para la evaluación de la posición en sentido bucolingual se recomienda utilizar $2 \mathrm{o}$ más exposiciones en diferentes planos del espacio utilizando alguna técnica de localización como la de Clark.

Las telerradiografías laterales y frontales pueden ayudar en la determinación de la posición del canino, determinando su relación con otras estructuras faciales como seno maxilar y piso de nariz. Las radiografías panorámicas son también usadas para localizar el canino impactado en los tres planos del espacio.

En el presente reporte se muestra los resultados del tratamiento exitoso de un caso poco frecuente de impactación bilateral de caninos superiores que había producido reabsorción de incisivos centrales. Se enfatiza la importancia de un diagnóstico clínico y radiográfico adecuado, así como la necesidad de establecer un plan de tratamiento integral que considere los aspectos estéticos y funcionales, sí como el manejo multidisciplinario del caso.

\section{Referencias}

1. Sapp JP, Eversole LR, Wysocki GP. Patología oral y máxilofacial contemporánea. España: Harcourt Brace, 1998:5.

2. Kuftinec M, Shapira S. The impacted maxillary canine: I Review of concepts. ASDC J Dent Child 1995; 62(5): 317-24.

3. Ballester JF, Toubia F, Irigoyen L. E canino incluido: diagnóstico y tratamiento. 1 ra ed. Editora MDB. España 1990.

4. Bishara SE. Impactad maxillary canines: a review. Am J Orthod Dentofacial Orthop 1992; 101(2): 159-71.

5. Bishara SE, Kommer DD, McNeil MH, Montagano LN, Oesterle LJ, Yoongquist HW. Management of impacted canines. Am J Orthod 1976; 69(4): 371-87.

6. Rimes RJ, Mitchell CN, Willmot DR. Maxillary incisor root resorption in relation to the ectopic canine: a review of 26 patients. Eur J Orthod 1997; 19(1): 79-84.

7. Ericson S, Kurol J. Radiographic assessment of maxillary canine eruption in children with clinical signs of eruption disturbances. Eur J Orthod 1988; 8(3): 133-40.

8. Fournier A, Turcotte JY, Bernard C. Orthodontic considerations in the treatment of maxillary impacted canines. Am J Orthod 1982; 81(3): 236-9.

9. Dewel BF. The upper cuspid: Its development and impaction. Angle Orthod 1949; 19: 79-90.

10. Jacoby $H$. The etiology of maxillary canine impactions. Am J Orthod 1983; 84(2):125-132.

11. Becker A, Bimstein E, Shteyer A. Interdisciplinary treatment of mul- tiple unerupted supernumerary teeth. Report of a case. Am J Orthod 1982; 81(5): 417-22.

12. Fastlicht $\mathrm{S}$. Treatment of impacted canines. Am J Orthod 1954; 40: 891-905.

13. Lewis PD. Preorthodontic surgery in the treatment of impacted canines. Am J Orthod 1971;60(4): 382-397.

14. Takahama T, Aiyama Y. Maxillary canine impaction as a posible microform of cleft lip and palate. Eur $\mathbf{J}$ Orthod 1982; 4: 275-277.

15. Becker A, Smith P, Behar R. The incidence of anomalous maxillary lateral incisors in relation to palatallydisplaced cuspids. Angle Orthod 1981;51(1): 24-29.

16. Langberg BJ, Peck S. Tooth-size reduction with occurrence of palatal displacement of canines. Angle Orthod 2000; 70(2): 126-28.

17. Brin I, Solomon Y, Zilberman Y. Trauma as a possible etiologic factor in maxillary canine impaction. Am J Orthod Dentofacial Orthop 1993; 104(2): 132-137.

18. Kaban LB. Cirugía bucal y máxilofacial en niños. Mexico: Editorial Interamericana; McGrae-Hill. 1992.

19. Becker A, Zilberman Y, Tsur B. Root length of lateral incisors adjacent to palatally-displaced maxillary cuspids. Angle Orthod 1984; 54(3): 218-25.

20. Brin I, Becker M, Shalhav M. Position of the maxillary permanent canine in relation to anomalous or missing lateral incisors: a population study. Eur J Orthod 1986; 8(1): 12-16.

21. Peck S, Peck L, Kataja M. The palatally displaced canine as a dental anomaly of genetic origin. Angle Orthod 1994; 64(4): 249-56.

22. Ericson S, Kurol J. Longitudinal study and analysis of clinical supervision of maxillary canine eruption. Community Dent Oral Epidemiol 1986; 14(3): 172-86.

23. Ericson S, Kurol J. Radiographic examination of ectopically erupting maxillary canines. Am J Orthod Dentofacial Orthop 1987; 91(6): 483-492. 